\title{
Fault current Characterization Based on Fuzzy Algorithm for DOCR Application
}

\author{
Luly Susilo, J.C. Gu, S.K. Huang \\ Electrical Engineering Department, National Taiwan University of Science and Technology. \\ Email: lulysusilo@gmail.com \\ Received January, 2013
}

\begin{abstract}
Penetration of distribution generation (DG) into power system might disturb the existing fault diagnosis system. The detection of fault, fault classification, and random changes of direction of fault current cannot always be monitored and determined via on-line by conventional fault diagnosis system due to DG penetration. In this paper, a fault current characterization which based on fuzzy logic algorithm (FLA) is proposed. Fault detection, fault classification, and fault current direction are extracted after processing the measurement result of three-phase line current. The ability of fault current characterization based on FLA is reflected in directional overcurrent relay (DOCR) model. The proposed DOCR model has been validated in microgrid test system simulation in Matlab environment. The simulation result showed accurate result for different fault location and type. The proposed DOCR model can operate as common protection device (PD) unit as well as unit to improve the effectiveness of existing fault diagnosis system when DG is present.
\end{abstract}

Keywords: Fault Current Characterization; Fuzzy Logic Algorithm; DOCR; Distributed Generation

\section{Introduction}

Penetration of DG that concentrated closer to customer side can improve the efficiency of electric power delivery. In the contrary, multi-source power system scheme as result of penetration of DG both in radial and ring power system might produce bi-directional power flow as well as fault current. The fault current may be in forward or reverse direction, and this will change rapidly depends on system requirement. It is inevitable; however, existing fault diagnosis system based on radial scheme with focusing only on single source can be no effective anymore. In conventional fault diagnosis system, after obtaining information operation status of protection device (PD) during/after fault event, then the information will be matched with table database in SCADA. Dispatcher will locate the fault section as soon as possible according to diagnosis result [1]. One aim of this work is to improve effectiveness of fault diagnosis system; PD will send its operating status to SCADA as well as the fault current characteristic information. Furthermore, dispatcher not only can locate the faulted section, but also accurately determine fault source and decide appropriate action in advance.

Sample of analog signal of line current will be processed in Digital Fourier Transform (DFT) module. Magnitude of phase line current, positive sequence current and zero sequence current as well as angle of positive sequence current will be taking into fuzzification input, from fuzzy interference system (FIS) that already constructed before, the membership value will be generated after comparing the input and membership function. Then from defuzzification process will decide the relationship of all input line current with related fault characterization information. Many publications already presented concept regarding fault detection, fault classification, and fault current direction which based on various intelligence control approaches (Wavelet, Neural Network, and FLA) $[2,4,7,8]$. Unfortunately, none of them clearly provided a complete concept fault characterization through a single approach. In this work, this solution is addressed. In part II, elaboration of FLA for fault characterization is provided in detail and systematic. The proposed microgrid test system as well as the DOCR and its control model in Matlab environment are shown in part III. Next part, brief discussion about simulation result is conducted before closing by conclusion part.

\section{Fuzzy logic algorithm (FLA) for fault current characterization}

The nature form of fault current characterization problem is not including complex mathematical expression. FLA is preferably to be implemented since this algorithm is simple and not involve any complex computation. Major procedures in this work are shown in Figure 1. 


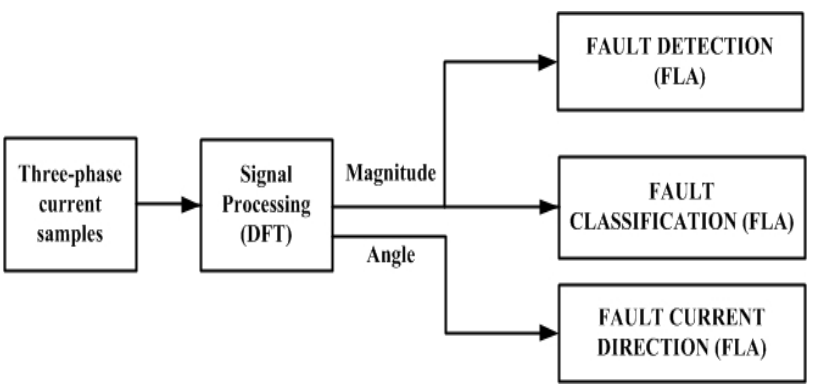

Figure 1. Fault current characterization block diagram.

\subsection{Fault Detection and Fault Classification}

Occurrence of shunt fault on power system create very low impedance path so very high fault current will appear and be delivered by power sources. Fault detection is important part in work in order to distinguish real fault current from transient/inrush current.

Transient/inrush period will generate significant amount of current, but it will decease very fast. Conventional DFT module is utilized to extract the magnitude of line current, magnitude of zero sequence current and angle of positive sequence current. Fault detection module will sense and record the current data both before and after 1 cycle at point where high current detected. If high current still remain within and after 1 cycle, it means fault is detected. Fault detection is denoted as $\delta_{0}$.

$$
\begin{gathered}
\delta_{0}=1 \quad \text { (fault), if I } \geqq \mathrm{I}_{\text {ref. }} . \\
\delta_{0}=0 \quad \text { (no-fault), else }
\end{gathered}
$$

where: $\mathrm{I}$ is measured line current and $\mathrm{I}_{\text {ref. }}$ is nominal line current.

The characteristic features of different types of fault are described in terms of $\delta_{1}, \delta_{2}, \delta_{3}$, and $\delta_{4}$ which are calculated as described below.

$$
\begin{gathered}
\delta_{1}=\frac{\mathrm{I}_{\mathrm{a}}-\mathrm{I}_{\mathrm{b}}}{\max \left(\mathrm{I}_{\mathrm{a}}, \mathrm{I}_{\mathrm{b}}, \mathrm{I}_{\mathrm{c}}\right)} ; \delta_{2}=\frac{\mathrm{I}_{\mathrm{b}}-\mathrm{I}_{\mathrm{c}}}{\max \left(\mathrm{I}_{\mathrm{a}}, \mathrm{I}_{\mathrm{b}}, \mathrm{I}_{\mathrm{c}}\right)} ; \\
\delta_{3}=\frac{\mathrm{I}_{\mathrm{c}}-\mathrm{I}_{\mathrm{a}}}{\max \left(\mathrm{I}_{\mathrm{a}}, \mathrm{I}_{\mathrm{b}}, \mathrm{I}_{\mathrm{c}}\right)} ; \delta_{4}=\frac{\mathrm{I}_{0}}{\mathrm{I}_{1}}
\end{gathered}
$$

where

$\mathrm{I}_{\mathrm{a}}, \mathrm{I}_{\mathrm{b}}, \mathrm{I}_{\mathrm{c}}$ are magnitudes of three phase current

$\mathrm{I}_{0}$ is zero sequence current

$\mathrm{I}_{1}$ is positive sequence current

Variation in fault location, power angle, fault inception angle and fault resistance are very important in order to study value of $\delta_{1}, \delta_{2}, \delta_{3}$, and $\delta_{4}$ for any kind of fault condition [2]. Hence two-bus power system as shown in Figure $\mathbf{2}$ is built to study the fault current characteristic features.

Line length $\mathrm{AB}$ is $60 \mathrm{kM}$, two sources $11.4 \mathrm{kV}$, frequency $60 \mathrm{~Hz}$ and with next following sequence impedance as tabulated in Table 1.
Fault location at $25 \%$ and $75 \%$ of line length; power angle for $10^{\circ}$ and $30^{\circ}$; fault inception angle at $0^{\circ}$ and 90 fault resistance $0.001 \Omega$ and $100 \Omega$; All these variations are considered in order to determine the fault current characteristic features $\left(\delta_{1}, \delta_{2}, \delta_{3}\right.$, and $\left.\delta_{4}\right)$ of the different types of fault. The result can be shown in Table 2 and summarized as following:

For $\delta_{1}, \delta_{2}$ and $\delta_{3}$, "high" means a value between 0.1 and 1, "medium" means a value between -0.15 and 0.4 , "low" means a value between -0.1 and -1 . For $\delta_{4}$, "high" means a value between 0.1 and 1 , "low" means a value between 0.01 and 0.015 . The triangular membership function of $\delta_{0}, \delta_{1}, \delta_{2}, \delta_{3}$, and $\delta_{4}$ are shown from Figure 3 to Figure 5.

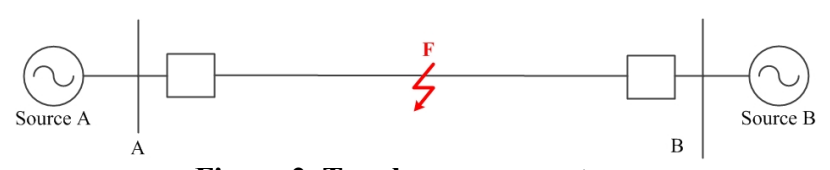

Figure 2. Two-bus power system.

\begin{tabular}{|c|c|c|c|c|c|}
\hline Fault Type & $\delta_{1}$ & $\delta_{2}$ & $\delta_{3}$ & $\delta_{4}$ & $\delta_{0}$ \\
\hline$a-g$ & high & medium & low & high & fault \\
\hline$b-g$ & low & high & medium & high & fault \\
\hline$c-g$ & medium & low & high & high & fault \\
\hline$a-b$ & medium & high & low & low & fault \\
\hline$b-c$ & low & medium & high & low & fault \\
\hline$c-a$ & high & low & medium & low & fault \\
\hline$a-b-g$ & medium & high & low & high & fault \\
\hline$b-c-g$ & low & medium & high & high & fault \\
\hline$c-a-g$ & high & low & medium & high & fault \\
\hline$a-b-c$ & medium & medium & medium & low & fault \\
\hline
\end{tabular}

Table 1. Impedance data of two-bus power system

\begin{tabular}{cccc}
\hline Sequence & $\mathrm{R}(\Omega / \mathrm{km})$ & $\mathrm{L}(\mathrm{mH} / \mathrm{km})$ & $\mathrm{C}(\mu \mathrm{F} / \mathrm{km})$ \\
\hline Zero & 0.38640 & 4.1264 & 7.751 \\
Positive & 0.01273 & 0.9337 & 12.74 \\
\hline
\end{tabular}

Table 2. Fuzzy rule of fault classification.

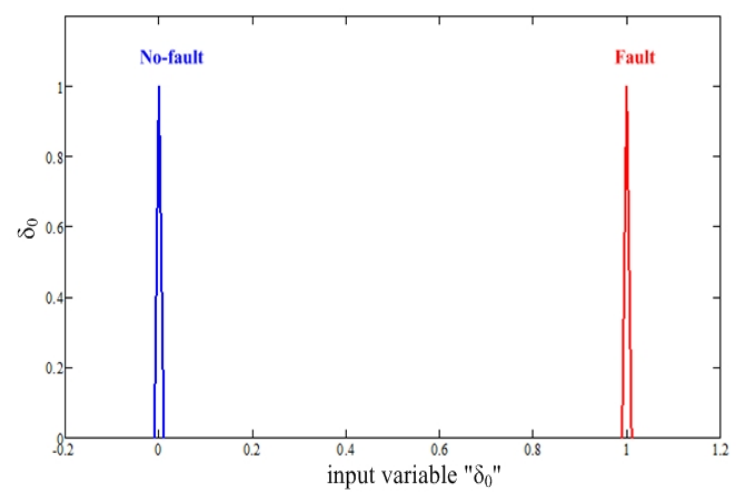

Figure 3. Fault detection triangular membership function. 


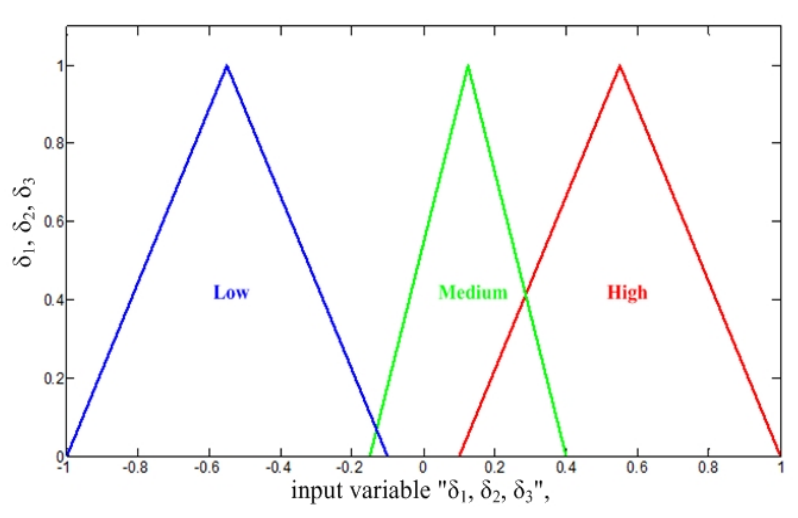

Figure 4. Fault classification triangular membership function for $\delta_{1}, \delta_{2}$, and $\delta_{3}$.

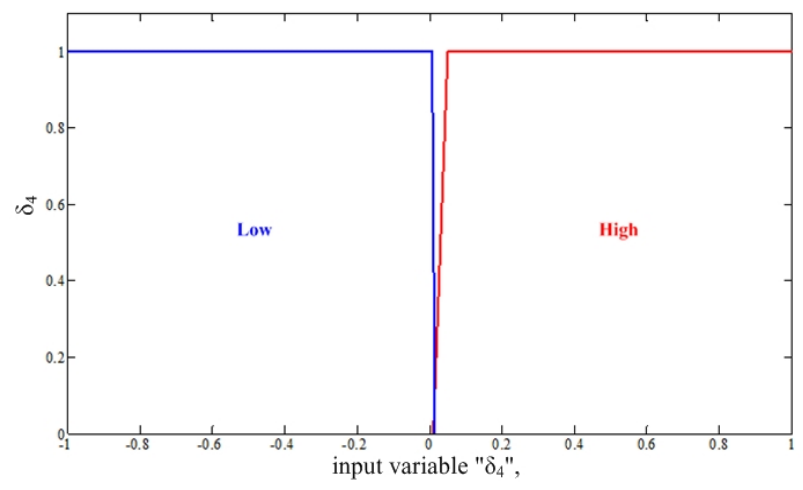

Figure 5. Fault classification triangular membership function for $\delta_{4}$.

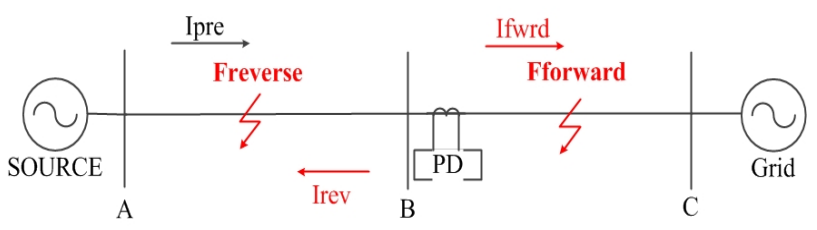

Figure 6. Forward fault and reverse fault.

\subsection{Fault Current Direction}

The original concept of fault current direction estimation can be found in reference $[3,4,5,6,9,10]$. The difference in angle of positive sequence between fault current and pre-fault current can be used to estimate the fault direction [3]. The pre-fault current is flow from source to grid as shown in Figure 6.

When fault forward occurs, the total fault current seen by PD is accumulation both pre-fault current and forward fault current. On the opposite, when fault reverse occurs, the total fault current seen by PD is subtraction between pre-fault current and reverse fault current. It is concluded that if the phase angle change value is negative that means forward fault occurs. On the contrary, if the phase angle change value is positive that means reverse fault occurs. The characteristic feature of fault direction is determined in terms of $\delta_{5}$, which calculated as following.

$$
\delta_{5}=\theta_{\text {Ipostfault }}-\theta_{\text {Iprefault }}
$$

where:

$\theta_{\text {Ipostfault }}$ is angle of postfault current

$\theta_{\text {Iprefault }}$ is angle of prefault current

As in previous section, the features for fault direction have been determined in terms of $\delta_{0}$ and $\delta_{5}$.

The fuzzy rule of fault current direction is determined as below:

If $\delta_{0}$ is " 1 " (fault) and $\delta_{5}$ is positive, it is reverse fault

If $\delta_{0}$ is " 1 "(fault) and $\delta_{5}$ is negative, it is forward fault

For $\delta_{5}$, "positive" means a value between $0^{\circ}$ and $180^{\circ}$, "negative" means a value between $0^{\circ}$ and $-180^{\circ}$. If the angle value is more than $180^{\circ}$, it shall be normalized with subtracting it with $360^{\circ}$. And if it less than $-180^{\circ}$, it shall be normalized with adding it with $360^{\circ}$ [3]. The triangular membership function can be seen in Figure 7.

\section{Test System and Directional Overcurrent Relay (DOCR) Model}

The proposed approach is validated on test system which shown in Figure 8. It consists of multisource power system including utility and several distributed generation sources, non-critical and critical load, and charging station. The system parameter of system in Figure 8 is tabulated in Table 3 .

\subsection{DOCR Model in Matlab Environment}

As shown in Figure 9, the DFT module will extract the magnitude of three phase line current, magnitude of positive sequence and zero sequence current and angle of positive sequence current. These values will be used as inputs for fault characterization module in order to compute the features characteristic $\left(\delta_{1} \sim \delta_{5}\right)$ of fuzzy logic module.

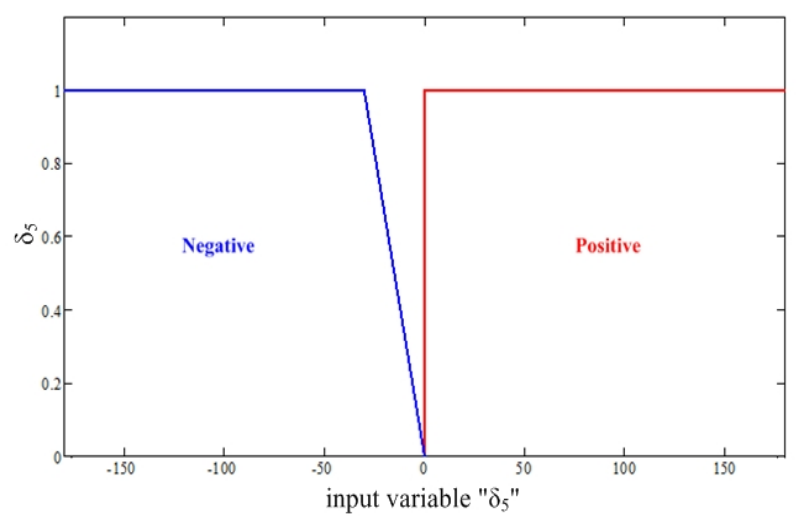

Figure 7. Fault current direction triangular membership function for $\boldsymbol{\delta}_{5}$. 


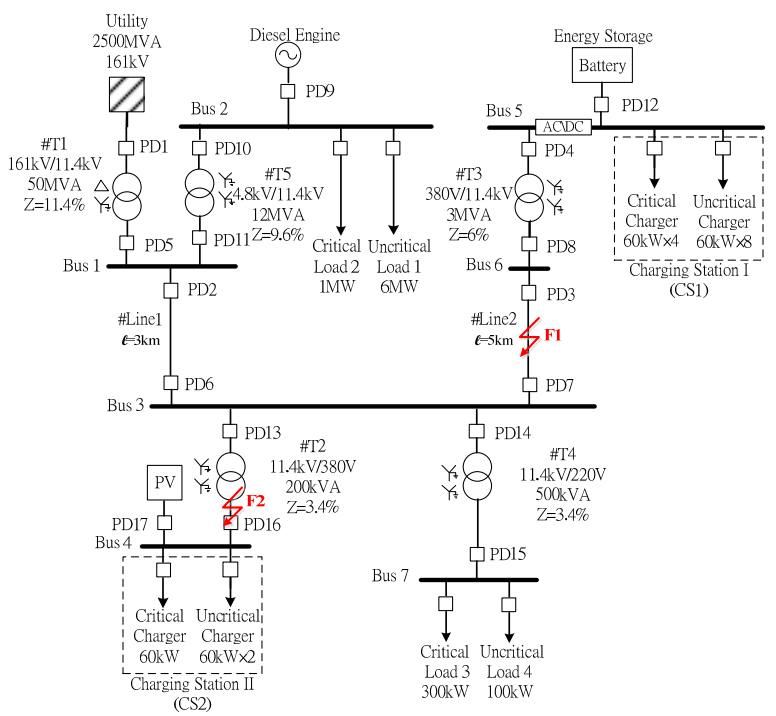

Figure 8. Test system.

Table 3. System parameter data for test system in Figure 8.

\begin{tabular}{|c|c|c|c|}
\hline Utility & \multicolumn{3}{|c|}{$\mathrm{MVA}_{\mathrm{sc}}=2500 \mathrm{MVA}, \mathrm{X} / \mathrm{R}=20 \%$} \\
\hline & $\mathrm{T} 1$ & \multicolumn{2}{|c|}{$50 \mathrm{MVA} 161 \mathrm{kV} / 11.4 \mathrm{kV} \mathrm{Z}=11.4 \%$} \\
\hline & $\mathrm{T} 2$ & \multicolumn{2}{|c|}{$300 \mathrm{kVA} 11.4 \mathrm{kV} / 380 \mathrm{~V} \mathrm{Z}=3.4 \%$} \\
\hline Transformer & $\mathrm{T} 3$ & \multicolumn{2}{|c|}{3 MVA $2.4 \mathrm{kV} / 11.4 \mathrm{kV} \mathrm{Z}=6 \%$} \\
\hline & $\mathrm{T} 4$ & \multicolumn{2}{|c|}{$500 \mathrm{kVA} 11.4 \mathrm{kV} / 380 \mathrm{~V} \mathrm{Z}=3.4 \%$} \\
\hline & $\mathrm{T} 5$ & \multicolumn{2}{|c|}{12 MVA 4.8 kV/11.4 kV Z=9.6\% } \\
\hline \multirow[b]{2}{*}{ \#Line } & 1 & $3 \mathrm{KM}$ & $\mathrm{R}_{1}=0.01273 \Omega / \mathrm{km}$ \\
\hline & 2 & $5 \mathrm{KM}$ & $\begin{array}{l}\mathrm{R}_{0}=0.38640 \Omega / \mathrm{km} \\
\mathrm{L}_{1}=0.9337 \times 10^{-3} \mathrm{H} / \mathrm{km} \\
\mathrm{L}_{0}=4.1264 \times 10^{-3} \mathrm{H} / \mathrm{km} \\
\mathrm{C}_{1}=12.740 \times 10^{-6} \mathrm{~F} / \mathrm{km} \\
\mathrm{C}_{0}=7.751 \times 10^{-6} \mathrm{~F} / \mathrm{km}\end{array}$ \\
\hline \multirow{3}{*}{$\begin{array}{l}\text { Distributed } \\
\text { Generation } \\
\text { (DG) }\end{array}$} & 1 & Diesel Engine & 3.125 MVA $4.8 \mathrm{kV}$ \\
\hline & 2 & PV & $150 \mathrm{kVA} 380 \mathrm{~V}$ \\
\hline & 3 & Energy Storage & $500 \mathrm{kVA} 380 \mathrm{~V}$ \\
\hline \multirow{8}{*}{ Load } & Load1 & Uncritical & $1 \mathrm{MW}$ \\
\hline & Load2 & Critical & $6 \mathrm{MW}$ \\
\hline & Load3 & Uncritical & $300 \mathrm{~kW}$ \\
\hline & Load4 & Critical & $100 \mathrm{~kW}$ \\
\hline & \multirow{4}{*}{$\begin{array}{l}\text { Charging } \\
\text { Station } \\
\text { (CS1) } \\
\text { Charging } \\
\text { Station } \\
\text { (CS2) }\end{array}$} & Uncritical & $60 \mathrm{~kW} \times 8$ \\
\hline & & Critical & $60 \mathrm{~kW} \times 4$ \\
\hline & & Uncritical & $60 \mathrm{~kW} \times 2$ \\
\hline & & Critical & $60 \mathrm{~kW} \times 1$ \\
\hline
\end{tabular}

In section III, as we already know that $\delta_{5}$ is difference between fault and pre-fault angle of positive current. The pre-fault positive current angle is obtained at 1 cycle before fault occurs and fault positive current angle is ob- tained at 1 cycle after fault occurs. The detail DOCR module can be seen in Figure 10.

\section{Simulation Result and Discussion}

For testing performance and accuracy of proposed approach, simulation was done in two fault locations in test system. They are marked as F1 and F2. Final results of fault characterization simulation of test system are tabulated in Table $\mathbf{4}$ and Table 5. Information regarding fault classification and fault current detection can be obtained accurately from this approach. Later, the information can be transferred through communication channel to SCADA for further fault diagnosis analysis. The proposed method in [2] use two different fuzzy rule base instead of combining become one fuzzy rule base as proposed in this work both for phase fault and ground fault. There fore, the proposed approach can work more effective.

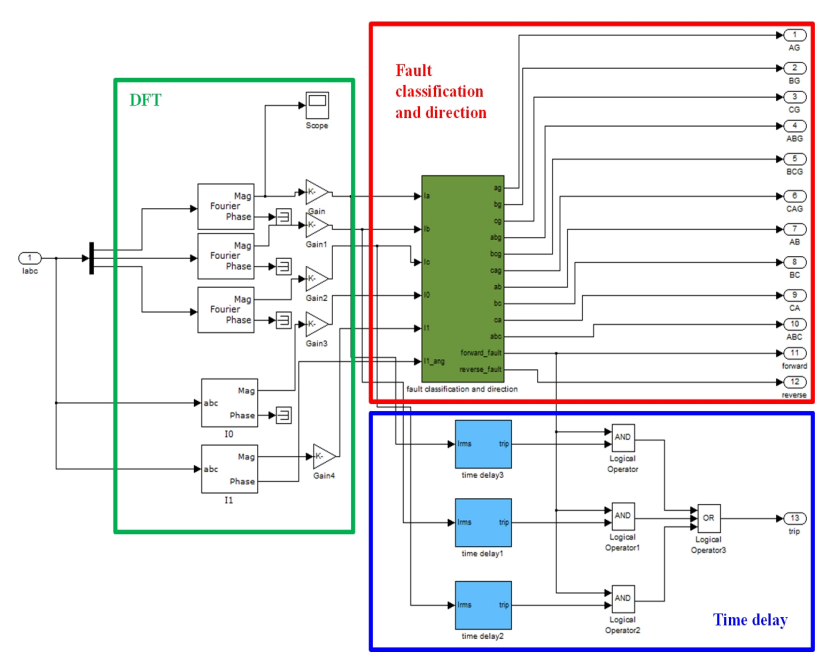

Figure 9. DOCR model in Matlab environment.

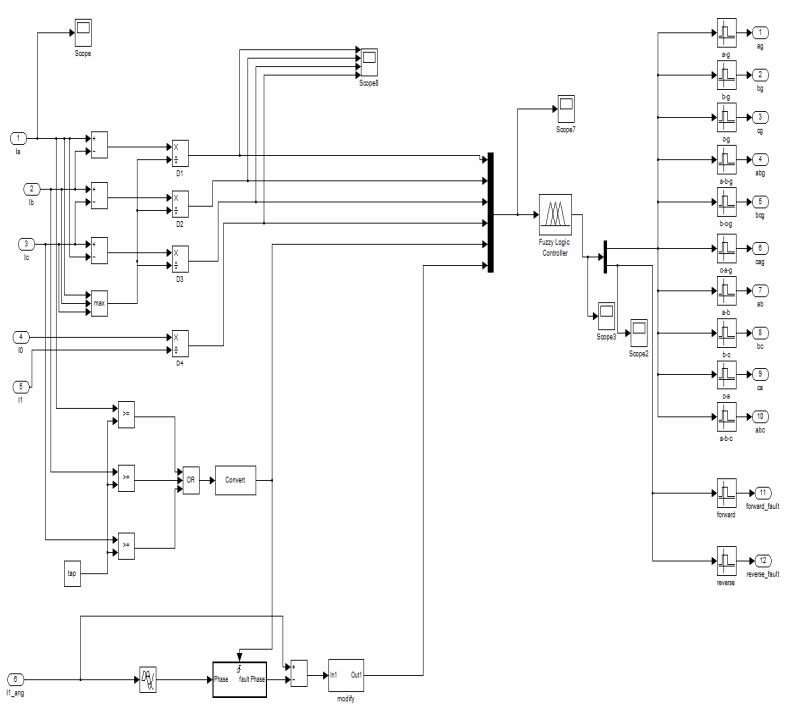

Figure 10. DOCR module for determining fault classification and fault current direction. 
Table 4. Simulation result for fault location at F1 (PD3 and PD7).

\begin{tabular}{ccccccccc}
\hline & & \multicolumn{6}{c}{ Fault information seen by PD } \\
\cline { 3 - 8 } $\begin{array}{c}\text { Fault } \\
\text { type }\end{array}$ & $\begin{array}{c}\text { Protection } \\
\text { Device } \\
\text { No. }\end{array}$ & & \multicolumn{2}{c}{ Fault type } & Fault direction & $\begin{array}{c}\text { Current Angle } \\
\text { (degree) }\end{array}$ \\
\cline { 3 - 8 } A-B-G & PD3 & $\checkmark$ & - & - & - & $\checkmark$ & 26.7 & 131.7 \\
& PD7 & $\checkmark$ & - & - & $\checkmark$ & - & 28.1 & -56.8 \\
& PD3 & - & $\checkmark$ & - & - & $\checkmark$ & 26.7 & 128.2 \\
B-C & PD7 & - & $\checkmark$ & - & $\checkmark$ & - & 28.1 & -57.9 \\
& PD3 & - & - & $\checkmark$ & - & $\checkmark$ & 26.7 & 138.3 \\
A-B-C & PD7 & - & - & $\checkmark$ & $\checkmark$ & - & 28.1 & -58.4 \\
\hline
\end{tabular}

Table 5. Simulation result for fault location at F2 (PD13 and PD16).

\begin{tabular}{|c|c|c|c|c|c|c|c|c|}
\hline \multirow{3}{*}{$\begin{array}{l}\text { Fault } \\
\text { type }\end{array}$} & \multirow{3}{*}{$\begin{array}{c}\text { Protection } \\
\text { Device } \\
\text { No. }\end{array}$} & \multicolumn{7}{|c|}{ Fault information seen by PD } \\
\hline & & \multicolumn{3}{|c|}{ Fault type } & \multicolumn{2}{|c|}{ Fault direction } & \multicolumn{2}{|c|}{$\begin{array}{l}\text { Current Angle } \\
\text { (degree) }\end{array}$} \\
\hline & & B-G & $\mathrm{B}-\mathrm{C}$ & A-B-C & Forward & Reverse & Pre-fault & Fault \\
\hline \multirow{2}{*}{ B-G } & PD13 & $\checkmark$ & - & - & $\checkmark$ & - & 25.9 & -46.4 \\
\hline & PD16 & $\checkmark$ & - & - & - & $\checkmark$ & 28.9 & 158.3 \\
\hline \multirow{2}{*}{$\mathrm{B}-\mathrm{C}$} & PD13 & - & $\checkmark$ & - & $\checkmark$ & - & 25.9 & -52.6 \\
\hline & PD16 & - & $\checkmark$ & - & - & $\checkmark$ & 28.9 & 164.6 \\
\hline \multirow{2}{*}{ A-B-C } & PD13 & - & - & $\checkmark$ & $\checkmark$ & - & 25.9 & -52.8 \\
\hline & PD16 & - & - & $\checkmark$ & - & $\checkmark$ & 28.9 & 171.4 \\
\hline
\end{tabular}

To detect the ground fault existence in system, the zero sequence current value has been considered. The detection of ground fault is denoted as $\delta_{4}$ in the proposed fuzzy logic scheme. The performance of proposed approach has also been studied for variation of operating conditions. The characteristic features value can be vary according to system parameter change and configuration. Any significant change can affect the fault current direction decision. Therefore, load flow study shall be performed at first in order to determine the normal current flow direction for pre-fault current reference.

\section{Conclusions}

An approach applying fuzzy logic algorithm (FLA) for fault current characterization was presented. The DOCR model based on this approach is developed in Matlab environment. DOCR model can operate and perform fault current characterization within 1 cycle after fault occurring. In addition to the FLA ability, not only fault detection was conducted; fault classification and fault current direction were also determined. Due to FLA has property to make decision in parallel, the whole process of fault current characterization take a very short time. The proposed DOCR model was applied to test power system and show accurate result as expected. Moreover, the proposed DOCR model can improve effectiveness of existing fault diagnosis system with delivering both its operating status and the fault current character information.

\section{REFERENCES}

[1] Y. Sekine, et al., "Fault Diagnosis of Power Systems," Proceedings of the IEEE, Vol. 80, No. 5, May 1992, pp. 673-683. doi:10.1109/5.137222

[2] R. N. Mahanty and P. B. Gupta Dutta, "A Fuzzy Logic Based Fault Classification Approach Using Current Samples Only," Electric Power Systems Research, 77, 2007, pp. 501-507.doi:10.1016/j.epsr.2006.04.009

[3] A. K. Pradhan, A. Routray and M. S. Gudipalli, "Fault Direction Estimation in Radial Distribution System Using Phase Change in Sequence Current," IEEE Transactions On Power Delivery, Vol. 22, No. 4, 2007, pp. 2065-2071.

[4] A. Ukil, B. Deck and V. H. Shah, "Current-Only Directional Overcurrent Relay," IEEE Sensor Journal, Vol. 11, No. 6, 2011, pp. 1403-1404. doi:10.1109/JSEN.2010.2094186

[5] A. K. Pradhan and P. Jena, "Solution to Close-in Fault Problem in Directional Relaying," IEEE Transactions On Power Delivery, Vol. 23, No. 3, 2008, pp. 1690-1692. doi:10.1109/TPWRD.2008.923149

[6] A. Ukil, B. Deck and V. H. Shah, "Smart Distribution Protection Using Current-only Directional Overcurrent Relay, IEEE PES Conference Innovative Smart Grid Technology, 2010, pp. 1-7.

[7] O. A. S. Youssef, "Fault Classification Based on Wavelet Transforms," IEEE PES T\&D Conference and Exposition, 2001, pp. 531-536.

[8] S. A. Gafoor and P. V. R. Rao, "Wavelet Based Fault Detection, Classification and Location in Transmission Lines," First International Power and Energy Conference-PECon, November 2006, pp. 114-118.

[9] S. Das, N. Karnik and S. Santoso, "Distribution Fault-Locating Algorithms Using Current Only," IEEE Trans. On Power Delivery, Vol. 27, No. 3, 2012, pp. 1144-1153. doi:10.1109/TPWRD.2012.2191422

[10] A. Ukil, B. Deck and V. H. Shah, "Current-Only Directional Overcurrent Protection for Distribution Automation: Challenges and Solutions," IEEE Transactions on Smart Grid, Vol. PP, No. 99, August 2012, pp. 1-8. 